\title{
Productor ejecutivo-creativo de series de ficción en la industria televisiva española
}

\author{
The executive-creative producer of fiction series in the Spanish \\ television industry
}

O produtor executivo-criativo de séries de ficção na indústria televisiva espanhola

María José Higueras Ruiz, Universidad de Granada, Granada, España (mhiguer@ugr.es) Francisco Javier Gómez Pérez, Universidad de Granada, Granada, España (frangomez@ugr.es)

Jordi Alberich Pascual, Universidad de Granada, Granada, España (jalberich@ugr.es)

RESUMEN | Este artículo tiene como objetivo analizar el protagonismo que adquiere el productor ejecutivo en el proceso de producción de series de ficción y su implicación en las facetas creativas. Con esta finalidad, se realiza una revisión del concepto de productor ejecutivo-creativo, así como de sus funciones y responsabilidades en cada fase del proceso. El trabajo se complementa con testimonios extraídos de once entrevistas a dieciséis destacados productores ejecutivos de la industria televisiva española realizadas durante el último trimestre del año 2017. A pesar de la variedad de modelos de actuación obtenidos, los resultados evidencian la relevancia activa y creciente de este profesional en los aspectos creativos de la producción televisiva española, así como la proyección internacional de sus trabajos, especialmente gracias a la implantación de las plataformas de streaming en este país.

PALABRAS CLAVE: productor ejecutivo; productor creativo; series de televisión; producción televisiva; showrunner; España; entrevistas.

Higueras-Ruiz, M. J., Gómez-Pérez, F. J., \& Alberich-Pascual, J. (2021). Productor ejecutivo-creativo de series de ficción en la industria televisiva española. Cuadernos.info, (50), 227-248.

https://doi.org/10.7764/cdi.50.27649 
ABSTRACT / This article analyses the role of the executive producer during the fiction TV series production process in Spain, with emphasis on the creative features. To do so, weused a qualitative methodology to review the executive-creative producer concept, as well as the duties and responsibilities developed by this professional in each phase of the production process. Additionally, the study is completed with testimonies from eleven interviews with sixteen executive producers in the contemporary Spanish television industry, which were developed during the last quarter of 2017. Despite the wide range of production models, the findings obtained suggest that the cited profile plays an active and growing role over contemporary Spanish TV series creativity. The international approach of these projects is noteworthy, especially thanks to the implantation of streaming platforms in this country.

KEYWORDS: executive producer; creative producer; TV series; television production; showrunner; Spain; interviews.

RESUMO|O presente artigo tem como objetivo analisar o protagonismo que o produtor executivo assume no processo de produção das séries de ficção e seu envolvimento com os aspectos criativos. Com essa finalidade, se realiza uma revisão do conceito de produtor executivo-criativo, assim como de suas funções e responsabilidades em cada fase do processo. Este trabalho complementa-se com testemunhas extraídas de onze entrevistas a dezesseis destacados produtores executivos da indústria televisiva espanhola realizadas durante o último trimestre de 2017. Apesar da variedade de modelos de atuação obtidos, os resultados evidenciam a relevância ativa e crescente deste profissional nos aspectos criativos da produção televisiva espanhola, bem como a projeção internacional de seus trabalhos, especialmente graças à implementação de plataformas de streaming no país.

PALAVRAS-CHAVE: produtor executivo; produtor criativo; séries de televisão; produção televisiva; showrunner; Espanha; entrevistas. 


\section{INTRODUCCIÓN}

En el siglo XXI, la tendencia creciente a producir series de ficción televisiva propicia que el sector audiovisual apoye su modelo de negocio en ellas en países como España, el sexto del mundo en exportación de este tipo de proyectos. La industria audiovisual en el país ha alcanzado protagonismo. De acuerdo con el Ministerio de Industria, Comercio y Turismo (Situación de la Industria Audiovisual en España, n.d.), representa el $28 \%$ de las industrias culturales que, asimismo, aportan en su conjunto un 3,2\% al PIB nacional.

Entre las potencialidades del mercado audiovisual en España, el informe del PwC (2018) destaca el talento profesional y creativo de los trabajadores del medio y el posicionamiento internacional del contenido español, especialmente gracias a las plataformas digitales, señalando la previsión del incremento de 10,1\% del CAGR $^{1}$ en el segmento de video OTT (over-the-top) en 2022.

En este contexto, se promueve la atención hacia la figura del productor ejecutivocreativo por parte de la industria, la audiencia y la academia, debido al incremento cuantitativo y cualitativo de series de ficción y al protagonismo del perfil en su producción. En el ámbito estadounidense, surge el concepto de showrunner para designar a ese profesional y destacar su presencia en cada una de las fases del proceso, especialmente desde una perspectiva creativa, propiciando su implicación en el resultado estético de la obra (Higueras-Ruiz et al., 2018). En palabras de Cascajosa (2018b), "la emergencia del showrunner [...] ha sido uno de los desarrollos más relevantes en la televisión contemporánea. [...] El showrunner no es solo una posición industrial, sino también un papel clave para incrementar el prestigio de la ficción televisiva"2 (p.126).

A pesar de la dificultad de definir a este perfil en la industria española contemporánea, debido a su constante evolución y a la asignación inexacta que se hace del título en determinadas ocasiones, consideramos adecuado comprender y referirnos al productor ejecutivo como productor ejecutivo-creativo. Este es el último responsable de la serie de ficción, encargado de contratar y supervisar a los diferentes departamentos, que mantiene una comunicación fluida, tanto con "los distintos equipos, que deben trabajar de modo coordinado, ajustándose a los plazos y presupuestos previstos, y teniendo unidad de visión creativa para alcanzar el estándar de calidad prefijado" (Pardo, 2014, p. 63), como con el resto de agentes que conforman el mercado televisivo: las cadenas de televisión y las plataformas.

\section{Compound Annual Growth Rate (tasa compuesta de crecimiento anual).}

2. En el texto original: "The emergence of the showrunner [...] has been one of the more relevant developments in contemporary television. [...] the showrunner is not only an industrial position, but also a critical role in increasing the prestige of television fiction" (Cascajosa, 2018b, p. 126). Traducción propia. 
El protagonismo adquirido por ese profesional, especialmente gracias al surgimiento e implantación de los servicios OTT en España, que fomentan la asociación de los proyectos con su productor ejecutivo-creador y la promoción de estos a través de dichos profesionales, motiva y justifica su atención desde el ámbito académico. Frente a los numerosos trabajos que se focalizan en el contenido de las series, aquellos dirigidos al estudio de los procesos de producción y creación representan un número creciente, pero minoritario (Mateos-Pérez, 2021). Este texto se dirige a dicha cuestión, con un aporte original a los estudios de la producción de los medios que permita conocer las labores, competencias y responsabilidades del productor ejecutivo-creativo en España desde la perspectiva práctica de su actuación. Se busca contribuir a fortalecer la base teórica correspondiente, vislumbrando las particularidades de la producción televisiva española en la última década, cuando su "desarrollo se ha visto impulsado por factores económicos, tecnológicos y culturales tan distintos a un pasado cercano que bien puede hablarse de una revolución seriada de la ficción nacional" (Huerta Floriano, 2020, p. 15).

Este texto estudia el proceso de producción de series de ficción televisiva en España en la última década (2010-2020) desde la perspectiva del perfil del productor ejecutivo-creativo (showrunner), atendiendo a su posición y relaciones en el interior del tejido industrial del mercado televisivo contemporáneo. El objetivo principal es conocer y analizar las coordenadas en las que dicho profesional realiza su labor dentro del contex to español, considerando las novedades en las estrategias de producción de las plataformas OTT y las implicaciones creativas del productor y el resultado estético de la ficción que crea y produce.

\section{MARCO TEÓRICO}

El perfil y concepto de productor ejecutivo destaca por su complejidad, tanto desde una perspectiva académica como profesional, pudiendo englobar diferentes tipologías y competencias en función de la empresa productora y del tejido industrial del país donde desarrolla su labor, así como del propio proyecto audiovisual (Diego, 2005). Sin embargo, independientemente de los factores señalados, existe una serie de características que se asocian con dicho crédito y permiten definirlo como "el principal responsable del control creativo y económico del programa [que] debe ser un hábil empresario y también poseer un grado elevado de sensibilidad artística" (Medina de la Viña \& Moreno Díaz, 2020, pp. 41-43). El productor ejecutivo es el encargado de aplicar los denominados estándares de producción: “los parámetros creativos, técnicos y de organización que caracterizan los contenidos de ficción desde el punto de vista de la producción, y que tienen que ver con alguna de las tres variables: tiempo, coste y calidad" (Diego \& Pardo, 2008, p. 62). 
En la historia de la ficción televisiva española advertimos tres periodos que denotan un carácter evolutivo y de constante cambio en la producción de esos proyectos, apreciando en todos los casos un componente creativo en la figura del productor. Un primer momento, de monopolio de Televisión Española, en el que se desarrolla una producción propia, de carácter autoral y liderada por productoresautores con labores de dirección y escritura de guiones. Un segundo periodo, de industrialización del sector debido a la implantación de las cadenas privadas y la cesión de la producción a empresas externas e independientes (García de Castro, 2002). Y una tercera etapa protagonizada por los avances de la digitalización, destacando la implantación de las plataformas OTT, y apreciando las variantes en la producción, distribución y consumo de las series de ficción y el protagonismo creciente de la figura del showrunner en dicho proceso (Cascajosa, 2019).

Las labores e implicaciones creativas asociadas al productor hallan su origen en el medio cinematográfico, donde destacan profesionales como el británico David Puttman que "se implica creativamente en cada una de sus películas, hasta el punto de ser tan responsable del resultado final como el director. Así, es denominado productor creativo o productor autor" (Pardo, 2001, p. 117).

Volviendo al terreno de la ficción televisiva, el productor ejecutivo debe tener las siguientes características: conocimiento del medio, dotes ejecutivas, criterio de calidad, conocimiento técnico, artístico y creativo, y competencia administrativa (Medina de la Viña \& Moreno Díaz, 2020, pp. 41-43). A pesar de que la adaptación del concepto anglosajón executive producer no se produce con total simetría en el medio español, el productor ejecutivo debe responder igualmente a un doble perfil ejecutivo-creativo (García de Castro, 2002) para determinar el formato y ajustar el presupuesto a sus aspiraciones creativas desde la primera idea.

Este perfil puede adoptar, asimismo, el crédito y las funciones del director o del guionista, destacando las opciones creativas que ello supone (Kellison, 2009). En España, la combinación guionista-director-productor no es tan común como en la industria norteamericana, aunque "en la producción financiada española se está adoptando este esquema de trabajo, de forma que los coordinadores de los equipos creativos son también productores ejecutivos" (Medina de la Viña \& Moreno Díaz, 2020, p. 41).

En la asignación de labores y crédito de productor ejecutivo a los coordinadores de guion debemos reconocer el trabajo precursor en España desarrollado por la compañía Globomedia (Diego, 2005). Siguiendo esta línea, el productor ejecutivo que ocupa el primer puesto de la jerarquía del equipo -el showrunner- debe disponer de las facultades que le permitan supervisar el desarrollo de la idea y la escritura de los guiones (Cascajosa, 2016). 
El productor ejecutivo no siempre posee la idea original de la ficción, aunque el concepto de showrunner en ocasiones se utiliza como sinónimo de creador, y contribuye a la consideración del autor televisivo (De la Torre, 2016). En cualquier caso, estamos ante un trabajo colaborativo donde el productor delega en cada miembro del equipo, que contribuye al resultado final del proyecto bajo su supervisión y coordinación, de manera que se mantenga la coherencia y no se desvirtúe la línea establecida (Higueras-Ruiz et al., 2019). Por lo tanto, este profesional representa "el hilo conductor que da unidad al complejo proceso de producción que siguen todas las ficciones televisivas” (Diego, 2005, p. 9).

\section{Etapas de producción de series de ficción y competencias del productor ejecutivo}

En la fase del desarrollo de la idea del proyecto, el productor ejecutivo se encarga de decidir el formato de acuerdo con la audiencia y estilo del programa (Pardo, 2014). Igualmente, posee o gestiona los derechos de autor de la idea y prepara el material para realizar el pitch a la cadena (Collie, 2007).

En la reunión con los ejecutivos de la cadena se debe presentar la viabilidad de la propuesta a nivel creativo, técnico, comercial y legal (Pardo, 2014), y establecer los parámetros de producción, narrativos y estéticos que permitan desarrollar la serie de ficción atendiendo a la variedad de profesionales que trabajarán en ella (Barroso, 2002). Cuando la cadena o plataforma acepta el proyecto comienza la contratación de los diferentes equipos, dando lugar a la fase de preproducción. Junto con las labores que el productor ejecutivo desarrolla desde una perspectiva económica y organizativa (contratación, presupuestos, desglose del guion técnico, calendario de rodaje) (Kellison, 2009), la cuestión más interesante desde un punto de vista creativo es el desarrollo del guion.

Previo al inicio del rodaje conviene haber finalizado la escritura de los guiones de toda la temporada (Kellison, 2009), para que el productor pueda atender plenamente al resto de departamentos durante la fase de producción. En este estadio, el productor ejecutivo supervisa el rodaje, en el que idealmente estará presente o disponible para revisar los planos televisivos, así como solucionar cualquier problema o duda al respecto (Schihl, 1997). La relación con el director es esencial y, aunque "el productor tiene facultad, tanto desde el punto de vista estético como creativo, para aceptar o rechazar las tomas de vídeo que se hagan a lo largo de toda la obra" (Schihl, 1997, p. 69), forma parte y depende de un equipo.

En la etapa de posproducción se tratan tres aspectos fundamentales: montaje o edición, efectos visuales y sonorización. El productor ejecutivo también podrá implicarse en promover, distribuir y explotar comercialmente la obra producida, haciendo patente su influencia creativa (Pardo, 2014). 


\section{Tejido industrial del medio televisivo en España}

En la industria televisiva, el productor ejecutivo debe mantener una relación de entendimiento y buena comunicación con los ejecutivos de las cadenas, quienes finalmente deciden sobre la viabilidad del proyecto (Medina, 2015). En función del grado de implicación y las responsabilidades técnicas, humanas, financieras y creativas adquiridas por cada agente se establece una modalidad de producción, destacando la asociada, en la que la cadena encarga el desarrollo del proyecto a una empresa productora, designa un productor asociado y aporta el financiamiento necesario (Ortiz, 2018). Actualmente, las cadenas de televisión funcionan como conglomerados multimedia que aglutinan diversos sectores de comunicación (Corporación RTVE, Atresmedia y Mediaset). Esto se debe a "la entrada en el mercado de novedosos y poderosos agentes tecnológicos y el cambio en los hábitos de consumo de cultura de los ciudadanos" (Pérez-Rufí, 2020, p. 240).

En este panorama sobresale la implantación de las plataformas OTT en el mercado televisivo español, como Netflix, Amazon Prime Video, HBO España, Disney+, Movistar+ o Filmin, incorporándose a la denominada streaming wars (Neira, 2020). Para profundizar en estos aspectos, consideramos la predominancia del servicio de Netflix (Heredia Ruiz, 2017), que desde el año 2018 cuenta con una sede de producción en Madrid, España, como centro neurálgico de la compañía a nivel europeo (Lacalle et al., 2019), y de la plataforma española de Movistar+, entre aquellas que han establecido alianzas para ofrecer sus servicios a la audiencia de forma conjunta y económica (Castro \& Cascajosa, 2020). Blakey (2017) describe una serie de cambios en los procesos de creación y producción de series de ficción, en su contenido y estética. Los tiempos de producción son más ágiles que en las cadenas generalistas, dando lugar a la estrategia denominada por Netflix straight-to-series: no producen un capítulo piloto y aseguran un presupuesto real para la temporada completa (Carrillo-Bernal, 2018). Ello supone incorporar simultáneamente al catálogo todos los capítulos de la temporada de las series propias y su posible visualización de una vez, promoviendo el efecto de atracón televisivo o binge-watching (Neira, 2020).

Las novedades más valoradas hacen referencia al formato, temática y estética de los proyectos, destacando una importante innovación creativa que está siendo adoptada por las cadenas generalistas (Huerta Floriano, 2020), que también ofrecen sus contenidos en portales en línea. Encontramos series de duración ajustada a los parámetros internacionales, dirigidas a un público de nicho, y con un marcado estilo cinematográfico, en ocasiones gracias a la incorporación de directores provenientes de dicho medio, especialmente en Movistar+ (Cascajosa, 2018a), con altos presupuestos asignados a sus proyectos originales ${ }^{3}$.

3. Series como La Peste o La Unidad contaron con un presupuesto de 10 millones de euros. 
Estos servicios permiten la viralidad de series como La Casa de Papel, lo que, de acuerdo con García Martínez (2020), se debe precisamente a "Netflix y su círculo virtuoso de la globalización, que la han aupado como el producto de la cultura popular española más conocido mundialmente por millenials y seriéfilos" (p. 100).

\section{MATERIALES Y MÉTODOS}

En esta investigación se aplica una metodología cualitativa, consistente en una revisión bibliográfica discutida en el marco teórico y entrevistas en profundidad. Por un lado, se acude a trabajos nacionales e internacionales especializados en la producción de proyectos audiovisuales de ficción televisiva: las características del productor ejecutivo creativo (Pardo, 2009, 2014), las funciones del perfil en cada fase del proceso de producción (Collie, 2007; Kellison, 2009; Marzal-Felici \& López-Cantos, 2008), la estructura industrial del medio televisivo (Medina, 2015; Medina de la Viña \& Moreno Díaz, 2020) y el panorama digital (Carrillo-Bernal, 2018; Cascajosa, 2018a, 2019; Neira, 2020).

Esta revisión bibliográfica permite sintetizar las labores del productor ejecutivocreativo en el contexto televisivo, de acuerdo con las funciones aplicadas por ese profesional en cada fase del proceso de producción y a las dos categorías que definen la dualidad del perfil: ejecutiva y creativa. Los resultados se presentan según el carácter económico-organizativo (tabla 1) o creativo (tabla 2) de esas funciones y las etapas establecidas.

\begin{tabular}{|c|c|c|}
\hline Fase & Etapa & Tareas \\
\hline $\begin{array}{l}\text { Diseño del } \\
\text { producto y } \\
\text { estudio de } \\
\text { mercado }\end{array}$ & $\begin{array}{l}\text { Desarrollo del } \\
\text { proyecto: } \\
\text { la idea }\end{array}$ & $\begin{array}{l}\text { - Elegir perfil o formato del proyecto } \\
\text { - Estudiar el mercado de las cadenas de TV } \\
\text { - Gestionar derechos de autor de la idea original } \\
\text { - Gestionar el presupuesto disponible }\end{array}$ \\
\hline \multirow{3}{*}{$\begin{array}{c}\text { Producción o } \\
\text { fabricación }\end{array}$} & $\begin{array}{l}\text { Preproducción: } \\
\text { el guion }\end{array}$ & $\begin{array}{c}\text { - Contratar al equipo humano, técnico, creativo } \\
\text { - Gestionar seguros y licencias } \\
\text { - Supervisar el presupuesto establecido }\end{array}$ \\
\hline & $\begin{array}{l}\text { Producción: } \\
\text { el rodaje }\end{array}$ & $\begin{array}{l}\text { - Controlar el plan de trabajo } \\
\text { - Gestionar recursos y aspectos de la producción } \\
\text { - Seguimiento del presupuesto establecido }\end{array}$ \\
\hline & $\begin{array}{l}\text { Posproducción: } \\
\text { la edición }\end{array}$ & $\begin{array}{l}\text { - Supervisar la edición: montaje, efectos visuales y } \\
\text { sonorización } \\
\text { - Controlar el presupuesto y plazos acordados }\end{array}$ \\
\hline Comercialización & $\begin{array}{l}\text { Promoción y } \\
\text { distribución: } \\
\text { la campaña } \\
\text { promocional }\end{array}$ & $\begin{array}{l}\text { - Balance: tiempo, coste y calidad } \\
\text { - Implicación durante la exhibición y distribución } \\
\text { - Intervención en la explotación comercial }\end{array}$ \\
\hline
\end{tabular}

Tabla 1. Síntesis de las tareas económico-organizativas del productor ejecutivo en el medio televisivo

Fuente: Higueras-Ruiz (2020), con base en Marzal-Felici \& López-Cantos (2008), Kellison (2009), \& Pardo (2014). 


\begin{tabular}{|c|c|c|}
\hline Fase & Etapa & Tareas \\
\hline $\begin{array}{l}\text { Diseño del } \\
\text { producto y } \\
\text { estudio de } \\
\text { mercado }\end{array}$ & $\begin{array}{l}\text { Desarrollo del } \\
\text { proyecto: } \\
\text { la idea }\end{array}$ & $\begin{array}{c}\text { - Elaborar el proyecto-propuesta } \\
\text { - Establecer los parámetros económicos, técnicos y } \\
\text { creativos } \\
\text { - Realizar el pitch a la cadena }\end{array}$ \\
\hline \multirow{3}{*}{$\begin{array}{l}\text { Producción o } \\
\text { fabricación }\end{array}$} & $\begin{array}{l}\text { Preproducción: } \\
\text { el guion }\end{array}$ & $\begin{array}{c}\text { - Intervenir en desarrollo del guion } \\
\text { - Control de la relación: guion-presupuesto } \\
\text { - Supervisar casting, decorados, localizaciones, vestuario, } \\
\text { maquillaje ... }\end{array}$ \\
\hline & $\begin{array}{l}\text { Producción: } \\
\text { el rodaje }\end{array}$ & $\begin{array}{l}\text { - Supervisión o presencia en el rodaje } \\
\text { - Gestionar problemas y situaciones críticas } \\
\text { - Solucionar preguntas referidas al guion }\end{array}$ \\
\hline & $\begin{array}{l}\text { Posproducción: } \\
\text { la edición }\end{array}$ & $\begin{array}{c}\text { - Intervenir en el montaje del pre-master: valoraciones y } \\
\text { cambios } \\
\text { - Propuestas de sonorización y música } \\
\text { - Control de calidad creativa }\end{array}$ \\
\hline Comercialización & $\begin{array}{l}\text { Promoción y } \\
\text { distribución: } \\
\text { la campaña } \\
\text { promocional }\end{array}$ & $\begin{array}{l}\text { - Supervisar contenidos creativos de campañas } \\
\text { publicitarias, merchandising, plataformas de distribución ... }\end{array}$ \\
\hline
\end{tabular}

Tabla 2. Síntesis de las tareas creativas del productor ejecutivo en el medio televisivo

Fuente: Higueras-Ruiz (2020), con base en Marzal-Felici \& López-Cantos (2008), Kellison (2009), \& Pardo (2014).

Los datos obtenidos de la revisión bibliográfica han sido complementados con los resultados de 11 entrevistas en profundidad realizadas durante el último trimestre del año 2017 a 16 destacados productores ejecutivos de series de ficción españolas. Los factores de selección de la muestra de informantes se hallan estrechamente asociados con las series de ficción que producen, indicando su último trabajo como referencia para obtener una muestra representativa de acuerdo a tres criterios principales:

1. El desarrollo del perfil de productor/a ejecutivo/a en el proceso de producción de la serie, y su correspondiente crédito, valorando asimismo la asignación de la creación original del proyecto.

2. La selección de al menos un profesional (o pareja profesional) que haya trabajado en una de las televisiones lineales, pública (TVE) o privadas (Antena3, Telecinco) que operan en España o en las dos plataformas OTT con mayor producción propia en ficción televisiva y número de suscriptores establecidas plenamente en dicho país: Netflix $\left(\mathrm{SVOD}^{4}\right)$ y Movistar+ (IPTV5) (Barlovento Comunicación, 2020).

4. Video bajo demanda por suscripción.

5. Televisión por protocolo de Internet. 
3. La actuación predominante del profesional durante la última década (20102020), siendo los proyectos producidos o emitidos en dicho periodo.

Asimismo, atendemos a la variedad de género (drama y comedia) de las series de ficción producidas, y de género (masculino y femenino) de los profesionales seleccionados. Con dichos criterios de inclusión se buscó alcanzar una muestra representativa para vislumbrar las diferencias halladas en la actuación del productor ejecutivo, especialmente en las plataformas OTT. La tabla 3 presenta la muestra de profesionales ordenados alfabéticamente.

La entrevista es ampliamente utilizada en los estudios sobre producción en los medios para conocer la actuación de los profesionales de la industria audiovisual, así como su relación con los diferentes agentes y contextos que intervienen en el proceso de producción (Bruun, 2016). Permite obtener "citas directas de los entrevistados acerca de sus experiencias, opiniones, sentimientos y conocimientos"6 (Patton, 1988 , p. 7). En este caso se aplicó una entrevista cualitativa, flexible, abierta y semiestructurada (Flick, 2012). Se elaboró una guía previa, a partir de la revisión bibliográfica (tabla 4), aunque se aceptaron nuevos temas durante la entrevista.

\begin{tabular}{cccc} 
Nombre & TV Series & Género & Cadena \\
\hline David Abajo \& David Fernández & Ella es tu padre (2017) & Comedia & Telecinco \\
\hline Olatz Arroyo \& Marta Sánchez & Supernormal (2021) & Comedia & Movistar+ \\
\hline Pablo Barrera & Brigada Costa del Sol (2019) & Drama & Telecinco \\
\hline Ramón Campos & Alta Mar (2019) & Drama & Netflix \\
\hline Juan Carlos Cueto \& & Toy Boy (2019) & Drama & Antena 3 \\
\hline Verónica Fernández & Hache (2019- ) & Drama & Netflix \\
\hline Darío Madrona \& Carlos Montero & Élite (2018-) & Drama & Netflix \\
\hline Pilar Nadal & Águila Roja (2009-2016) & Drama & TVE \\
\hline Javier Olivares & El Ministerio del Tiempo (2015-2020) & Drama & TVE \\
\hline Álex Pina & White Lines (2020) & Drama & Netflix \\
\hline Alberto \& Jorge Sánchez Cabezudo & La Zona (2017) & Drama & Movistar+ \\
\hline
\end{tabular}

Tabla 3. Profesionales entrevistados y producciones a su cargo

Fuente: Elaboración propia.

6. En el texto original: "Direct quotations from people about their experiences, opinions, feelings, and knowledge" (Patton, 1988, p. 7). Traducción propia. 
¿Quién es el showrunner? ¿Qué habilidades y facultades desarrolla?

¿Cuál es su posición en el organigrama de la empresa productora? ¿Es factible el modelo de producción asociado a la figura del showrunner o resulta utópico en la práctica?

¿Cuándo surge este perfil? ¿Ha existido siempre?

Bloque I:

El showrunner como productor eje cutivo-creativo
Bloque II:

El proceso de producción de series de ficción
Bloque III:

El panorama de la televisión en España

\section{Preproducción:}

¿Cuáles son las funciones del showrunner en el departamento de guion?

¿Es apropiada la figura del guionista-productor? ¿Cómo podría contribuir al éxito del programa?

¿Cómo es la dinámica de trabajo en el departamento de guion?

¿Cuál es la relación del showrunner con otros guionistas?

¿Cómo se reparten los créditos de la escritura de guion?

¿Qué otras labores desarrolla el showrunner durante la preproducción?

\section{Producción:}

¿Cuáles son las labores del showrunner durante el rodaje?

¿Está presente?

¿Cómo es su relación con el director del capítulo?

\section{Posproducción:}

¿Cuáles son las labores del showrunner durante la posproducción?

¿Cómo es la relación del showrunner con el editor?

¿Quién toma la decisión sobre el final cut?

Según los teóricos nos hallamos inmersos en la Tercera Edad Dorada de la Televisión ¿es esto cierto? ¿en qué podemos basarnos?

¿Cómo influye la digitalización en el mercado televisivo en España?

¿Cómo es la relación del showrunner con la cadena?

¿Pueden los ejecutivos de la cadena cambiar la idea original del proyecto?

¿Cómo influyen los ejecutivos de la cadena en la creatividad de la obra?

¿Qué cambios acontecen en la industria televisiva como consecuencia de la implantación de plataformas OTT en España?

\section{Tabla 4. Guía de la entrevista}

Fuente: Elaboración propia. 
Las preguntas se clasifican en tres bloques: 1) El showrunner como productor ejecutivo-creativo, 2) El proceso de producción de series de ficción, y 3) el panorama de la televisión en España. Se incluyeron cuestiones introductorias de carácter biográfico, a pesar de una revisión curricular previa de cada profesional, para iniciar la sesión creando un ambiente cómodo, y otras de conclusión para sintetizar y cerrar el encuentro. El esquema de preguntas puede variar cuando el perfil y trayectoria profesional del entrevistado motivan a ceder una mayor atención a ciertas preguntas frente a otras.

Durante los encuentros, los profesionales autorizaron la grabación del audio de la sesión, así como el uso de los datos de forma no anónima en el contexto de esta investigación. La posterior transcripción del audio facilita la interpretación y la extracción de los testimonios pertinentes en cada caso. Para ello, se analizan las respuestas en función de los bloques temáticos indicados, comparando la información y definiendo los resultados que articulan el discurso.

\section{RESULTADOS}

\section{El showrunner como productor ejecutivo-creativo}

Como se ha mencionado en el marco teórico, el productor ejecutivo debe responder a un doble perfil ejecutivo-creativo para determinar el formato y ajustar el presupuesto a sus aspiraciones creativas desde la primera idea. Según Juan Carlos Cueto ${ }^{7}$, productor ejecutivo, junto con Rocío Martínez, de Mar de Plástico (Antena 3, 2015-2016) o Toy Boy (Antena 3, 2019), "cuando empiezas a escribir ya sabes, por ejemplo, el porcentaje de exteriores que tendrás pactado con la dirección de producción". Siguiendo esta idea, Pablo Barrera ${ }^{8}$, productor ejecutivo de Brigada Costa del Sol (Telecinco, 2019), afirma que este profesional "tiene que ser una figura, que no existen muchas, que aúne lo artístico, lo creativo, y, al mismo tiempo, tenga capacidad para gestionar lo puramente material".

Con el fin de asegurar los niveles de calidad y ajuste presupuestario del proyecto, el productor debe supervisar el trabajo realizado en los diferentes equipos. Así lo expresa Álex Pina', productor ejecutivo de series como La Casa de Papel (Antena 3, 2017-2018; Netflix, 2019-2020) o El Embarcadero (Movistar+, 2019-2020): “los productores ejecutivos estamos en guion, pero también estamos en la sala de montaje, en el casting, y en todas las reuniones que son importantes para que la serie tenga unas determinadas características, es decir, un diferencial".

7. Juan Carlos Cueto y Rocío Martínez, entrevistados por el autor, 16 de noviembre y 22 de noviembre de 2017.

8. Pablo Barrera, entrevistado por el autor, 10 de octubre de 2017.

9. Álex Pina, entrevistado por el autor, 23 de noviembre de 2017. 
Por otra parte, a modo de ejemplo de la combinación guionista-directorproductor (Kellison, 2009), en España los productores de Crematorio (Canal+ 1, 2011) y La Zona (Movistar+, 2017), Jorge y Alberto Sánchez-Cabezudo ${ }^{10}$, combinan ambos perfiles y sus responsabilidades. En este punto, Juan Carlos Cueto añade que suele trabajar con equipos de tres personas para tomar todas las decisiones: "una de plató, que está más con actores y en el día a día del rodaje; y otras dos que están más pendientes de los guiones".

Los productores ejecutivos que han trabajado en Globomedia aprecian el sistema de ascenso hacia mayores responsabilidades creativas, basado en la formación práctica, la experiencia profesional y la confianza. Se trata de adaptar el "modelo norteamericano, en el cual desde hacía años ya se le daba importancia al productor creativo", añade Juan Carlos Cueto.

Respecto de la relación guionista-director-productor, y las facultades de supervisar el desarrollo de la idea y escritura de guiones, Ramón Campos ${ }^{11}$, cofundador de Bambú Producciones junto con Teresa Fernández-Valdés, y productor ejecutivo de Las Chicas del Cable (Netflix, 2017-2018) o Fariña (Antena 3, 2018), expone que "ser buen guionista no quiere decir ser buen productor ejecutivo, igual que un buen productor ejecutivo no tiene porqué ser un buen guionista, hay muchas partes que unir y si las consigues todas tienes un buen productor ejecutivo". Del mismo modo, Javier Olivares ${ }^{12}$, productor ejecutivo de la serie El Ministerio del Tiempo (TVE, 2015-2020), expresa que "un showrunner puede ser productor, guionista o director. Lo importante es que, sea guionista o no, esté en el embrión de una idea", y así indica que "la profesión más creativa de este negocio no es ni la del guionista ni la del director, es la del productor".

Es frecuente que el productor ejecutivo provenga del departamento de guion, no obstante, "su poder dependerá del peso que tenga dentro de la productora", estando bajo la supervisión del dueño de esta, aporta David Fernández ${ }^{13}$, productor ejecutivo junto con David Abajo de series de ficción como Ella es tu Padre (Telecinco, 2017). Ante dicha casuística, en la última década se han creado pequeñas compañías productoras lideradas por un guionista-productor que posee la libertad creativa y financiera para desarrollar proyectos con un marcado carácter personal. Entre otros, destacamos el caso de Javier Olivares, fundador de Cliffhanger, que anota

10. Jorge y Alberto Sánchez-Cabezudo, entrevistados por el autor, 11 de diciembre de 2017.

11. Ramón Campos, entrevistado por el autor, 4 de diciembre de 2017.

12. Javier Olivares, entrevistado por el autor, 17 de octubre de 2017.

13. David Fernández y David Abajo, entrevistado por el autor, 28 de noviembre y 22 de diciembre de 2017. 
que estamos ante "una fórmula inventada que te permite la creatividad", o de Álex Pina, creador de Vancouver Media, que añade: “Tener tu propia compañía te permite salvaguardar tu sello, tu mirada y tu ADN. Entonces haces lo que quieres y creces al ritmo que quieres".

\section{El proceso de producción de series de ficción}

Los entrevistados coinciden con que existen diferentes opciones para vender el proyecto: un documento de breve extensión (Alberto y Jorge Sánchez-Cabezudo), un flyer de una única página (Ramón Campos), o el guion del capítulo piloto (David Fernández y David Abajo).

En la fase de preproducción y guion, el productor ejecutivo contrata a los guionistas que trabajarán bajo su supervisión y con las indicaciones de la cadena. En este punto, hallamos diferentes formas y grados de su implicación en el departamento de guion. A modo de ejemplo, Álex Pina interviene en la realización de las tramas y escaletas, realizando la edición final junto con la productora y guionista Esther Martínez Lobato. Del mismo modo, Javier Olivares supervisa las escaletas y cada versión del guion, encargándose de la escritura. Por su parte, Juan Carlos Cueto y Rocío Martínez destacan la pertinencia de que el productor ejecutivo escriba el capítulo piloto, ya que les permite mostrar el tono y el estilo de la serie, convirtiéndose en referencia para el resto del equipo.

En esta fase es especialmente relevante que el productor ejecutivo establezca buenas relaciones con los profesionales de la cadena. Ramón Campos destaca la buena aceptación de las notas que reciben, pues considera que estamos ante "un trabajo de equipo, desde el origen hasta el final, y en ese equipo está la cadena". De manera similar, Álex Pina expresa: "Yo intento siempre trabajar las notas de la cadena, hacerlas, y cuando estoy en contra de algo busco una tercera vía alternativa”. Por lo tanto, "el entendimiento con la cadena es esencial, porque tienen en su mano paralizar el rodaje y si no ven la secuencia también tienes que entenderles", añade Pilar Nadal ${ }^{14}$, productora ejecutiva de Águila Roja (TVE, 2009-2016).

En cuanto al rodaje, el desarrollo del proceso pone de manifiesto que no siempre se escriben todos los guiones previamente y, en el contexto convencional español, "el presupuesto no te permite planificar todo para poder estar siempre en el momento exacto", indica Javier Olivares. Sin embargo, en las producciones para plataformas OTT es más probable tener todos los textos cerrados antes del rodaje, lo que hace posible trabajar cinematográficamente, es decir, por decorados naturales. Así ocurrió en la producción de La Zona o de La Peste (Alberto Rodríguez y Rafael Cobos, Movistar+, 2017).

14. Pilar Nadal, entrevistada por el autor, 1 de diciembre de 2017. 
Sobre la presencia del productor en el set, Ramón Campos afirma: "Yo estoy en rodaje las dos o tres primeras semanas para marcar el tono de cámara”; después, ejerce dicho control mediante la visualización diaria de las secuencias grabadas. Por su parte, Álex Pina, a excepción de momentos clave, no suele estar presente en el set, aunque existe una comunicación constante con el director y reuniones previas para trabajar el guion. En todo caso, el productor ejecutivo debe responder a las preguntas que surjan, ya que "tiene la visión y tiene que transmitir esa idea, porque es muy fácil que las cosas se vayan desviando, no por mala intención, sino porque cada uno puede interpretarlo de una manera", explica Rocío Martínez. En palabras de Ramón Campos, "el productor ejecutivo debe tener cabeza creativa, tener claro cuál es la 'foto' que quiere conseguir, y llevar a todo el equipo hasta esa 'foto'".

En este contexto también es importante recordar la pertinencia de no redirigir al director, como lo señala Javier Olivares: "El director tiene que mandar en plató, y si hay algo que no me gusta, en privado se lo puedo decir".

En la fase de posproducción, el productor y el director disponen de todo el metraje grabado y sus documentos asociados, y trabajan con dicho material junto con el editor, considerando las indicaciones realizadas por la cadena, apunta David Abajo.

No obstante, también existen diferentes grados de intervención sobre la edición. Ramón Campos trabaja activamente en la posproducción, remontando junto con el profesional encargado el material recibido. Por su parte, los hermanos SánchezCabezudo destacan el trabajo de efectos especiales delegado a una empresa externa y valoran su actuación en pareja: “Jorge estaba más en montaje, y yo con la música, el sonido y el etalonaje”, añade Alberto. Profesionales como Darío Madrona ${ }^{15}$, productor ejecutivo de Élite (2018-) junto con Carlos Montero, aprecian un incremento en el grado de implicación en esta fase que, en todo caso, dependerá del contrato firmado con la cadena o plataforma: "Es verdad que en la parte de posproducción en mi caso sí he notado que cada vez he ido trabajando más en ella, sobre todo en montaje".

En último lugar, en la mayoría de los casos los profesionales afirman que las funciones de promoción, distribución y explotación comercial son lideradas por los departamentos de marketing de la cadena o plataforma de emisión.

\section{El panorama de la televisión en España}

En cuanto al panorama actual de la televisión en España, si atendemos a los testimonios de los entrevistados, destacamos la actitud positiva con la que en su mayoría lo acogen. Verónica Fernández ${ }^{16}$, productora ejecutiva de Hache (2019- )

15. Darío Madrona y Carlos Montero, entrevistados por el autor, 28 de noviembre y 4 de diciembre de 2017.

16. Verónica Fernández, entrevistada por el autor, 8 de noviembre de 2017. 
para Netflix, destaca algunas ventajas, como más oferta de trabajo, libertades creativas y posibilidades de globalización:

Hay proyectos que teníamos en el cajón que han salido a la luz y se están leyendo. Por otra parte, puedes plantear cosas que en un determinado momento la televisión generalista no quería y que estas nuevas plataformas se atreven a hacer y, luego, lo que yo creo que es lo más importante, la internacionalización.

En relación con el proceso de producción, encontramos un modelo que fomenta la responsabilidad asignada al productor ejecutivo de los proyectos. Así lo señala Olatz Arroyo ${ }^{17}$, productora ejecutiva y creadora, junto con Marta Sánchez, de Supernormal (2021-): "Movistar quiere que haya alguien capitaneando, que tenga clara la idea y luche para que todos los equipos trabajen a favor de esta. Asimismo, incidimos en la aplicación de los estándares internacionales para los formatos televisivos y en las variantes en la narrativa y estética de los proyectos". En este sentido, los productores ejecutivos de Élite (2018- ) valoran la flexibilidad de Netflix en la duración variable de los capítulos de la serie.

Finalmente, nos dirigimos a las figuras de Ramón Campos y Álex Pina, por el carácter pionero de su actuación ejecutiva-creativa y la potente repercusión internacional de sus producciones gracias a las plataformas OTT. Ramón Campos firma la primera serie original de producción española de Movistar+ (Velvet Colección, 2017-2019) y de Netflix (Las chicas del cable, 2017-2019). El creador incide en la meta internacional de sus trabajos y el estilo cinematográfico, y sigue dos líneas temáticas distintas: melodrama romántico con misterio y thriller policiaco, apreciando su mayor implicación en esta última.

Por su parte, Álex Pina también ha trabajado para ambos operadores: El embarcadero (Movistar+, 2019-2020) y White Lines (Netflix, 2020). Destacamos la firma del contrato de exclusividad entre el creador y Netflix (Cascajosa, 2019), y la renovación de La Casa de Papel, la serie de habla no inglesa más vista de la plataforma y ganadora del premio Emmy internacional a mejor drama en 2018. La evolución de los personajes, atractivos para el espectador a pesar de ser antihéroes, responde a uno de los factores más sobresalientes y representativos del trabajo de Álex Pina.

En las producciones de ambos showrunners podemos apreciar una factura visual, estructura dramática, líneas temáticas, y equipo técnico y casting reiterativos, que derivan de sus decisiones y configuran un sello característico, creativo y personal en las series de ficción de cada uno de estos profesionales, motivando la reflexión sobre su asignación autoral en el medio.

17. Olatz Arroyo y Marta Sánchez, entrevistadas por el autor, 7 de noviembre y 11 de octubre de 2017. 


\section{DISCUSIÓN Y CONCLUSIONES}

Los resultados de la investigación nos ubican en un panorama complejo, que evoluciona constantemente dentro de la industria televisiva en general y del medio español en particular, donde las posibilidades de la digitalización propician una serie de cambios en la producción y características de las series de ficción. En tal contexto, la figura del productor ejecutivo-creativo experimenta un protagonismo creciente.

Aunque atribuir responsabilidades creativas a este perfil en España no supone una novedad, constatamos su predominancia en los procesos de producción, que permite vincularlo con el concepto anglosajón de showrunner. Junto con las labores de planificación y gestión económica, el análisis de las funciones desarrolladas por el productor ejecutivo nos permite indicar un conjunto significativo de responsabilidades creativas que desempeña a lo largo de las diferentes fases del proceso de creación y producción de series de ficción.

Este profesional participa en el surgimiento y desarrollo de la idea desde su origen, estableciendo una serie de parámetros que configuran el formato del proyecto audiovisual. Posteriormente, se hace patente su intervención en el equipo de guion, a través de la supervisión de los textos, las indicaciones de cambios y, especialmente, la reescritura de la última versión. Durante el rodaje, atiende a las dudas y soluciona los problemas que surgen en el set, donde idealmente estará presente. Finalmente, el productor ejecutivo supervisa la edición, participando activamente en ella o mediante notas.

Los productores ejecutivos entrevistados dentro de la industria española revelan un perfil altamente creativo, destacando las labores que desarrollan sobre dichos aspectos, especialmente en el departamento de guion, de donde procede la mayoría de ellos. Así, se consideran entes creativos, lo que los asemeja al modelo imperante en la industria norteamericana.

Por otra parte, los testimonios ponen de manifiesto que, a pesar de poseer la máxima responsabilidad en dicho proceso, los productores ejecutivos trabajan dentro de un medio colaborativo, donde deben asegurar la integridad y continuidad de la obra. Es imprescindible que los aportes realizados por los diferentes departamentos se adapten a los parámetros establecidos, para que no se desvirtúe la idea original y se respete la visión del/los creador/es. Por lo tanto, el trabajo en equipo y las relaciones dentro de él son esenciales para adquirir un producto de calidad, junto con las indicaciones de los ejecutivos de la cadena o plataforma que financia y distribuye el proyecto. 
En este contexto, es esencial considerar la implantación de las plataformas OTT en el sector televisivo español, por cuanto han contribuido a la producción de contenidos novedosos y atractivos desde una perspectiva formal, temática y estética y, asimismo, han motivado modificaciones análogas en las cadenas generalistas. Los profesionales entrevistados valoran positivamente dichas opciones, apreciando una mayor atención hacia su trabajo en los procesos de creación y desarrollo de estos proyectos.

Finalmente, insistiendo en el carácter cambiante del medio televisivo contemporáneo, las diferencias en el modo de producción y resultados de diferentes proyectos y la inclusión de nuevos agentes en la industria de la televisión, insistimos en la pertinencia de complementar esta investigación con futuros estudios.

\section{FINANCIAMIENTO}

Este artículo ha sido escrito durante la contratación pre-doctoral: "Formación de Profesorado Universitario" (FPU 15/00737) del Gobierno de España. Proyecto de Investigación de I+D de Plan Nacional «Transmedialización y crowdsourcing en las narrativas de ficción y no ficción audiovisuales, periodísticas, dramáticas y literarias» (20182020) (Ref. CSO2017-85965-P) Domingo Sánchez-Mesa Martínez y Jordi Alberich-Pascual. Estancia de Investigación financiada por el Plan Propio de la Universidad de Granada en la Universidad Rey Juan Carlos de Madrid, supervisada por el Dr. Alfonso Palazón Meseguer. Agradecemos a los profesionales por aceptar la entrevista y ofrecer datos de valor para esta investigación.

\section{REFERENCIAS}

Barlovento Comunicación. (2020). Análisis de la industria televisiva-audiovisual (Analysis of the television-audiovisual industry). Retrieved from https://www.barloventocomunicacion.es/ wp-content/uploads/2020/12/analisis-industria-televisiva-y-audiovisual-2020-BLV.pdf

Barroso, J. (2002). Realización de los géneros televisivos (Production of television genres). Síntesis.

Blakey, E. (2017). Showrunner as Auteur: Bridging the Culture/Economy Binary in Digital Hollywood. Open Cultural Studies, 1, 321-332. https://doi.org/10.1515/culture-2017-0029

Bruun, H. (2016). The Qualitative Interview in Media Production Studies. In C. Paterson, D. Lee, A. Saha, \& A. Zoellner (Eds.), Advancing Media Production Research: Shifting Sites, Methods, and Politics (pp. 131-146). Palgrave Macmillan. 
Carrillo-Bernal, J. (2018). Paradigma Netflix. El entretenimiento de algoritmo (The Netflix Paradigm. Algorithm Entertainment). Editorial UOC.

Cascajosa, C. (2016). El ascenso de los showrunners: Creación y prestigio crítico en la televisión contemporánea (The Rise of Showrunners: Creation and Critical Prestige in Contemporary Television). Index comunicación, 6(2), 23-40. Retrieved from

https://journals.sfu.ca/indexcomunicacion/index.php/indexcomunicacion/article/view/255

Cascajosa, C. (2018a). De la televisión de pago al video bajo demanda. Análisis de la primera temporada de la estrategia de producción original de ficción de Movistar+ (From PayTV to Video-on-Demand. An Analysis of the First Season of Movistar+'s Strategy of Original Fiction Production). Fonseca, Journal of Communication, (17), 57-74. https://doi.org/10.14201/fjc2018175774

Cascajosa, C. (2018b). The emergence of the showrunner in global contemporary TV fiction: The case of Pau Freixas in Catalonia. Catalan Journal of Communication \& Cultural Studies, 10(1), 125-132. https://doi.org/10.1386/cjcs.10.1.125_1

Cascajosa, C. (2019). Producción propia en las televisiones españolas. Modelos tradicionales y servicios VOD (Own Production on Spanish Television. Traditional Models and VOD Services). In C. F. Heredero (Ed.), Industria del cine y el audiovisual en España. Estado de la cuestión. 2015-2018 (Film and Audiovisual Industry in Spain. State of the Art. 2015-2018) (pp. 181-218). Caimán Cuadernos de Cine y Festival de Málaga.

Castro, D. \& Cascajosa, C. (2020). From Netflix to Movistar+: How Subscription Video-onDemand Services Have Transformed Spanish TV Production. Journal of Cinema and Media Studies, 59(3), 154-160. https://doi.org/10.1353/cj.2020.0019

Collie, C. (2007). The Business of TV Production. Cambridge University Press.

De la Torre, T. (2016). Historia de las series (TV Series History). Roca Editorial.

Diego, P. (2005). La figura del productor de ficción en televisión (The Profile of the Fiction Producer in Television). Communication and Society, 18(1), 9-30. https://bit.ly/3xbNjGG

Diego, P. \& Pardo, A. (2008). Estándares de producción de “dramedias” familiares en España: El caso de Médico de familia, Cuéntame cómo pasó y Los Serrano (Production Standars of Family "Dramedies" in Spain: The case of Médico de familia, Cuéntame cómo pasó and Los Serrano). In M. Medina (Ed.), Series de televisión (Television Series) (pp. 45-74). Yumelia.

Flick, U. (2012). Introducción a la investigación cualitativa (3d ed.) (An Introduction to Qualitative Research). Ediciones Morata.

García de Castro, M. (2002). La ficción televisiva popular. Una evolución de las series de televisión en España (Popular Television Fiction. An Evolution of Television Series in Spain). Gedisa.

García Martínez, A. N. (2020). La Casa de Papel: entre el atraco perfecto y la telenovela partisana (Money Heist: Between the Perfect Robbery and Partisan Soap Opera). In M. A., Huerta Floriano (Ed.), Revolución seriada. El gran cambio de la ficción televisiva en España (TV Series Revolution. The Great Change of Television Fiction in Spain) (pp. 99-122). Tirant Humanidades. 
Heredia Ruiz, V. (2017). Revolución Netflix: desafíos para la industria audiovisual (Netflix Revolution: challenges for the audiovisual industry). Chasqui. Revista Latinoamericana de Comunicación, (135), 275-295. https://doi.org/10.16921/chasqui.v0i135.2776

Higueras-Ruiz, M. J., Gómez-Pérez, F. J., \& Alberich-Pascual, J. (2018). Historical Review and Contemporary Characterization of Showrunner as Professional Profile in TV Series Production: Traits, Skills, Competences, and Style. Communication and Society, 31(1), 91-105. Retrieved from

https://revistas.unav.edu/index.php/communication-and-society/article/view/35721

Higueras-Ruiz, M. J., Gómez-Pérez, F. J., \& Alberich-Pascual, J. (2019). Historical and Conceptual Review of Authorship and its Implications for Television Media: The Concept of Author in American Contemporary Fiction TV-Series. Doxa Comunicación, 28, 79-96.

\section{https://doi.org/10.31921/doxacom.n28a04}

Higueras-Ruiz, M. J. (2020). La producción ejecutiva-creativa de las series de ficción televisiva contemporánea: conceptualización, responsabilidades y dinámicas de autoría vinculadas al perfil audiovisual del showrunner (Executive-Creative Production of Contemporary Fiction TV Series: Conceptualization, Responsibilities and Authorship Associated with the Audiovisual Profile of Showurnner) (Doctoral dissertation, Universidad de Granada). Retrieved from http://hdl.handle.net/10481/65319

Huerta Floriano. (Ed.) (2020). Revolución seriada. El gran cambio de la ficción televisiva en España (TV Series Revolution. The Great Change of Television Fiction in Spain). Tirant Humanidades.

Kellison, C. (2009). Producing for TV and New Media: A Real-World Approach for Producers (2nd ed.). Focal Press.

Lacalle, C., Gómez, B., Sánchez, M., \& Pujol, C. (Eds.) (2019). Obitel 2019. Modelos de Distribución de la Televisión por Internet: Actores, Tecnologías, Estrategias (Obitel 2019. Internet Television Distribution Models: Actors, Technologies, Strategies). Editora Sulina.

Marzal-Felici, J. \& López-Cantos, F. (2008). Teoría y técnica de la producción audiovisual (Theory and Technique of Audiovisual Production). Tirant lo Blanch.

Mateos-Pérez, J. (2021). La investigación sobre series de televisión españolas de ficción. Un estudio de revisión crítica (1998-2020) (Research on Spanish fiction television series. A critical review study (1998-2020)). Revista Mediterránea de Comunicación, 12(1), 171-190. https://www.doi.org/10.14198/MEDCOM000016

Medina, M. (2015). Estructura y gestión de empresas audiovisuales (3d ed.) (Structure and Management of Audiovisual Companies). EUNSA.

Medina de la Viña, E. \& Moreno Díaz, J. (2020). La producción en televisión. Contexto, herramientas $y$ proceso (5th Ed.) (Television Production. Context, Tool and Process). Ommpress Bookcrafts.

Neira, E. (2020). Streaming Wars: La nueva televisión (Streaming Wars. New Television). Barcelona, Spain: Libros Cúpula.

Ortiz, M. J. (2018). Producción y realización en medios audiovisuales (Production and Filmmaking for Audiovisual Media). RUA Universidad de Alicante. 
Pardo, A. (2001). Historia y ficción en el cine de David Puttnam (History and Fiction in David Puttman's Films). Historia Contemporánea, 22 (2), 117-149. Retrieved from https://hdl.handle.net/10171/27542

Pardo, A. (2009). El productor creativo: ¿tautología o excepción? (Creative Producer: Tautology or Exception?). In J. Marzal-Felici \& F. Gómez-Tarín (Eds.), El productor y la producción en la industria cinematográfica (Producer and Film Industry Production) (pp. 45-66). : Universidad Complutense.

Pardo, A. (2014). Fundamentos de producción y gestión de proyectos audiovisuales (Bases for Producing and Managing Audiovisual Projects). EUNSA.

Patton, M. Q. (1988). How to Use Qualitative Methods in Evaluation (2nd ed.). SAGE.

Pérez-Rufí, J. P. (2020). Televisión en España y grupos mediáticos internacionales: políticas de comunicación europeas y alianzas frente a las plataformas VOD globales (Television in Spain and International Media Groups: European Communication Policies and Alliances against Global VOD Platforms). In A. Suing \& V. Kneipp (Eds.), Olhares sobre a imagen em movimiento (Looking at the moving image) (pp. 236-263). Ria Editorial.

PwC. (2018). Entertainment and Media Outlook 2018-2022 Spain. https://pwc.to/3AjhDBm

Rodríguez, A. (2018, March 1). Conferencia-diálogo con Alberto Rodríguez. Lecture given at the Jornadas de Orientación Profesional y Empleabilidad (Conferences of Career Guidance and Employability). Facultad de Comunicación y Documentación. Universidad de Granada, España.

Schihl, R. J. (1997). Dramáticos en televisión: Organización y procesos (Studio Drama: Processes and Procedures. Multiple Camera Video Series). M IORTV.

Situación de la Industria Audiovisual en España (Spanish Audiovisual Industry). (n.d.). Invest in Spain. Retrieved from https://www.investinspain.org/es/sectores/audiovisual 


\section{SOBRE LOS AUTORES}

MARÍA JOSÉ hIGUeRAS RUIZ, doctora en Ciencias Sociales por la Universidad de Granada, donde ejerce como profesora del Departamento de Información y Comunicación. Forma parte del equipo de investigación CommuniCAV "Procesos de creación, producción y postproducción audiovisual y multimedia" (código PAIDI SEJ-585) y del Proyecto de Investigación de Excelencia Transmedialización y crowdsourcing en las narrativas de ficción y no ficción audiovisuales, periodísticas, dramáticas y literarias (Ref. CSO2017-85965-P). Ha publicado artículos en destacadas revistas científicas internacionales, y participado en congresos sobre comunicación y producción audiovisual.

iD https://orcid.org/0000-0002-6849-3433

FRANCISCO JAVIER GómeZ PÉREZ, doctor en Comunicación Audiovisual por la Universidad de Sevilla, profesor del Departamento de Información y Comunicación de la Facultad de Comunicación y Documentación de la Universidad de Granada; miembro del equipo de investigación CommuniCAV "Procesos de creación, producción y postproducción audiovisual y multimedia" (código PAIDI SEJ585); coordinador de la monografía Políticas de impulso a las industrias audiovisuales (2015) y autor de Consolidación industrial del cine andaluz (2013).

iD https://orcid.org/0000-0001-7539-1681

JORDI ALBERICH PASCUAL, catedrático de Universidad en Comunicación Audiovisual y Publicidad, y subdirector del Departamento de Información y Comunicación de la Universidad de Granada. Es investigador principal del Grupo de investigación Procesos de creación, producción y postproducción audiovisual y multimedia (SEJ-585), del Plan Andaluz de Investigación. Es autor de cerca de medio centenar de publicaciones científicas de primer nivel, y ha desarrollado su actividad investigadora como experto en comunicación en los nuevos medios y en creación audiovisual colaborativa.

iD https://orcid.org/0000-0001-6871-4614 